\title{
Competencia del Terapeuta Ocupacional en el abordaje de la cultura organizacional ${ }^{1}$
}

\author{
Lina Nizeth Uribe Jiménez ${ }^{2}$, \\ Luz Elena Rodríguez ${ }^{3}$, \\ Marcy Viviana Acosta \\ Ana María Gómez
}

Si es cierto que la particularidad de un individuo se transparenta en su personalidad; la individualidad de una organización se manifiesta en su cultura.

Allaire y Firsirotu (1992)

\section{SÍNTESIS}

El proceso ocupacional de trabajo, se constituye en uno de los ámbitos de intervención profesional, que demanda del terapeuta ocupacional la utilización de sustentaciones teóricas para promover, mantener o mejorar el bienestar ocupacional.

El documento presenta el resultado de una investigación documental, cuyo objetivo consiste en determinar la competencia del terapeuta ocupacional en el abordaje de la cultura organizacional. Por su carácter, la metodología investigativa es descriptiva, donde la información revisada, se explica, interpreta y registra, para señalar los fundamentos conceptuales y prácticos de competencia del terapeuta ocupacional en la cultura empresarial, vista ésta, como uno de los factores para interpretar el comportamiento ocupacional de los trabajadores y orientar interdisciplinariamente las diferentes estrategias profesionales hacia la consecución de la salud y bienestar laboral en las organizaciones.

1 Trabajo de investigación para optar el título de terapeutas ocupacionales. Universidad Nacional. Agosto, 1999.

2 Terapeuta Ocupacional, Universidad Nacional de Colombia.

Terapeuta Ocupacional, Universidad Nacional.

Terapeuta Ocupacional, Universidad Nacional.

5 Terapeuta Ocupacional. Docente Universidad Nacional de Colombia. Directora trabajo de grado. 


\begin{abstract}
The work occupational process is a precinct of professional intervention that demand to occupational therapist the utilization theoreticals sustentions for promote, maintain or improve the occupational well-being.

The document present the result of a documental investigation, whose objective consist en determine the occupational therapist competence in the organizational culture approach. For it character, the investigative methodology is descriptive, where the revised information is explaining, interpreting and registering, for indicate conceptuals and practicals foundations of occupational therapist competence in the empresarial culture, see it as one of the factors for interpret the workers occupational behavior and interdisciplinary orientates the differents strategies toward the attainment of the health and laboral well-being in the organizations.
\end{abstract}

El artículo presenta una nueva forma de participación profesional del terapeuta ocupacional en las organizaciones o empresas, comprendiendo la cultura organizacional como medio para intervenir en el bienestar laboral del trabajador.

Se propone exponer este tema a la comunidad de terapia ocupacional y poner en consideración una nueva perspectiva de competencia profesional que amerita ser contemplada dentro del sector laboral particularmente.

Este documento es producto de una investigación de carácter documental, que a partir de marcos de referencia como el Modelo de la Ocupación Humana fundamenta la interdependencia persona y ambiente y por consiguiente al proceso ocupacional de trabajo como la interacción entre trabajador - empresa, poseedores de una cultura, que al ser abordada por el terapeuta ocupacional, le permite crear, promover $y$ desarrollar acciones para recuperar, mantener o promover el bienestar ocupacional de la persona dentro de su organización laboral.

Es así como se presentan algunos fundamentos conceptuales y práctiços de competencia profesional, con la pretensión de incentivar y mostrar a los profesionales otros campos de acción, factibles de desarrollar y a la vez, invitarlos a la interpretación y discusión sobre la pertinencia de desempeñarse profesionalmente en ellos. 


\section{CONCEPTUALIZACIÓN DE LA CULTURA}

La cultura se puede considerar como el conjunto de cualidades o formas de vida que define y regulan la relación cotidiana de los miembros de un grupo humano, a través de patrones de comportamiento que los caracterizan.

En la literatura de terapia ocupacional, diferentes autores comparten y otorgan diversos elementos a este concepto.

De acuerdo a Hocking y Whiteford (1995), la cultura es considerada el conjunto de normas y creencias de un grupo que regulan la esencia de los valores fundamentales en la práctica del terapeuta: igualdad, libertad, justicia, verdad, prudencia, dignidad y altruismo, para así enfrentarse al contexto en el cual trabaja.

Para Dyck y Forwell (1997), la influencia de la cultura se ha centrado en el compromiso con la ocupación y en la dinámica de los procesos terapéuticos, que considerada en diferentes contextos, permite apreciar los valores, creencias, experiencias, costumbres y patrones de comportamiento de ciertos grupos.

La importancia de interpretar la cultura en un contexto terapéutico le permite al terapeuta ocupacional conocer los antecedentes culturales de los usuarios como factores que influyen en las interacciones sociales y en los objetivos considerados para hacer efectiva y eficaz la intervención; en el favorecimiento de una buena comunicación; en el establecimiento de metas terapéuticas, teniendo en cuenta los valores e intereses $d$ los usuarios y la individualización de la terapia. (Mattingly y Beer, 1998).

El Modelo de Competencia Multicultural en Educación del Reino Unido, de Well (1996), citado por Mac Donald (1998), incorpora las áreas de Autoexploración, Conocimientos y Habilidades, como herramientas para que los estudiantes en sus prácticas, tengan en cuenta el aprendizaje de conceptos tales como raza, etnicidad y cultura de sus usuarios para implementar las acciones e intervenciones más indicadas.

En este sentido, Sharon (1999), identifica a raíz de su propia experiencia, diferentes elementos en los diversos escenarios del cuidado de la salud; refiriendo que si de abordar a la persona holísticamente se trata, es necesario que los practicantes co- 
nozcan los factores sociales y culturales que pueden influir en las modalidades de tratamiento, teniendo en cuenta el espectro de valores, creencias y experiencias de los usuarios que determinan en un momento dado la percepción que tienen de la actividad, la consideración temporal, el lenguaje y otros.

Lo anterior, permite determinar que la cultura como patrón general de conducta, valores, creencias, costumbres y normas de una sociedad o grupo en particular, repercute en el actuar cotidiano de las personas. Por tanto, es necesario conocer el entorno cultural del lugar de trabajo, los rasgos.culturales de quienes participan en dicho proceso e inclusive de aquellos a quienes se les ofrece un servicio, para así elegir las estrategias más indicadas de interacción, comunicación e intervención dado el caso.

De esta manera, al considerar que cualquier grupo que se desenvuelve en un contexto posee características que orientan șu desempeño y a la vez lo diferencian de otros, se puede concebir que toda organización, -entendida ésta como el conjunto de personas que dirigen sus acciones en pro de un objetivo-, cuentan con unos patrones de comportamiento determinados, que se hacen indispensables considerar, cuando se desea abordar, intervenir o hacer parte de dicho grupo.

Así, al abordar el proceso de trabajo como la resultante de la interacción de la persona con su empresa, se puede contemplar la cultura al interior de ésta, denominada Cultura Organizacional, que hace referencia al conjunto de características y estrategias efectivas de interacción, que cohesionan a los miembros de una organización y orientan su desempeño ocupacional.

\section{COMPETENCIA PROFESIONAL}

La competencia se refiere a la utilización de sustentaciones teóricas por parte de un grupo de profesionales, tomando como punto de partida el objeto de estudio de su propia disciplina, para determinar su intervención profesional en el abordaje de las dimensiones del ser humano, cuyo conocimiento y servicio llaman su atención. Trujillo (1987).

Por tanto, partiendo de la ocupación como objeto de estudio y con el ánimo de establecer la manera de participar profesionalmente en el área de la cultura 
organizacional, en el estudio se determinaron los fundamentos conceptuales y prácticos para que el terapeuta ocupacional ejerza profesionalmente. El cuadro No. 1 esquematiza la secuencia conceptual que permite comprender dicha competencia.

\section{COMPETENCIA DEL TERAPEUTA OCUPACIONAL EN EL ABORDAJE DE LA CULTURA ORGANIZACIONAL}

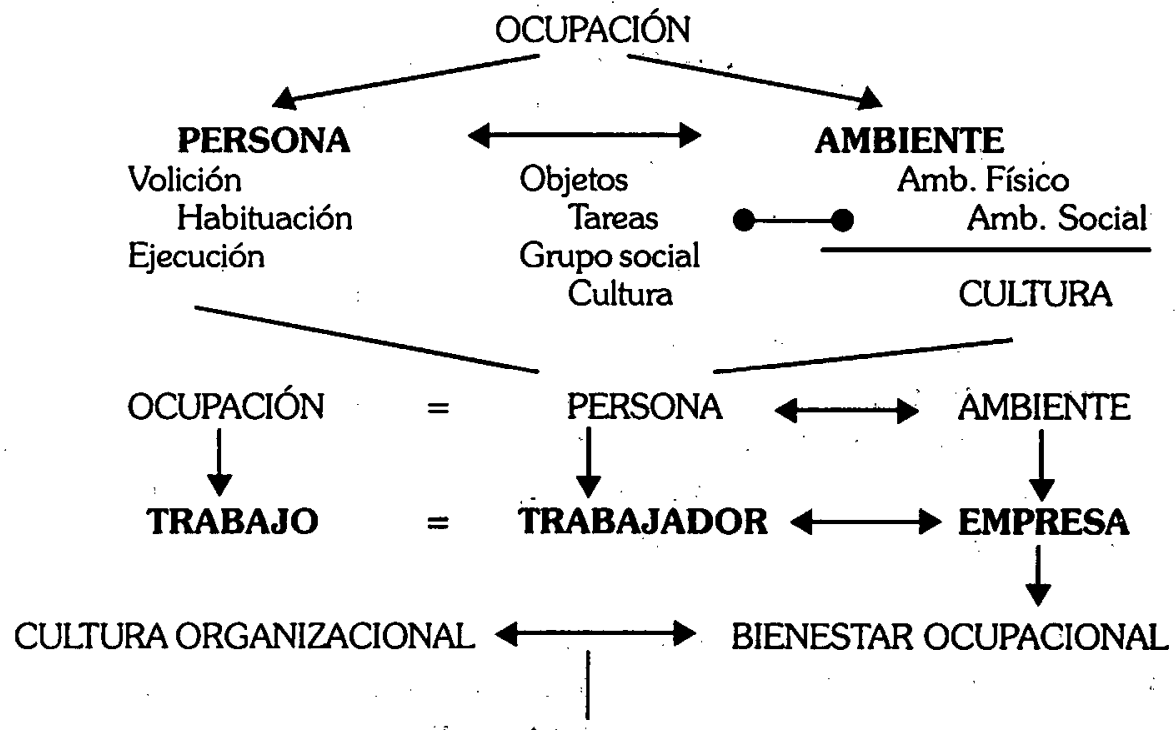

FORMACIÓN PROFESIONAL

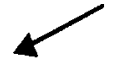

CONCEPTUAL

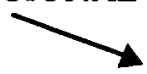

PRACTICA

Cuadro No. 1. Secuencia conceptual de la investigación.

La cultura organizacional: un factor que influye en el bienestar ocupacional del trabajador: La cultura de una organización o empresa se crea al determinarse o estipularse la misión, visión, normas y objetivos para el funcionamiento de ésta, partiendo de los valores, convicciones, costumbres y otros elementos constitutivos de la cultura de los empresarios y trabajadores, que se manifiestan en su vida laboral y se mantienen o modifican según expectativas propias y organizacionales y el grado de flexibilidad existente en las políticas. 
Los valores, normas, hábitos, creencias, roles, dirección estratégica y estructura organizacional son algunos de los elementos constitutivos de la cultura, que influyen en el bienestar laboral y éste a su vez repercute en la manera de fomentarse y arraigarse dicha cultura (ver cuadro No. 2). De lo contrario, si una cultura no favorece el trabajo en una organización, se generan efectos negativos tanto para el trabajador como para la empresa. (Ver cuadro No. 3).

El Terapeuta Ocupacional al tener conocimiento de la dinámica cultural de una empresa y sus efectos, puede llegar a un intento por comprender e interpretar sus manifestaciones y así, desarrollar acciones para optimizar, mantener y/o mejorar el ambiente laboral, objetivizado en el desempeño de cada persona y en los grupos en general. Por tanto, el terapeuta ocupacional valora formas de interacción social, cuidado personal del trabajador, responsabilidades laborales, entre otros para comprender el desempeño ocupacional del trabajador.

La intervención en la problemática de la cultura organizacional es una acción interprofesional en donde el terapeuta ocupacional puede formar parte de los equipos de trabajo, en áreas como la salud Ocupacional y de Recursos Humanos, a través de la prevención y control de riesgos psicosociales, adaptaciones al medio laboral, valoración de condiciones de trabajo, procesos de selección, inducción y capacitación, entre otros.

Así, para ejercer con propiedad en esta área, el terapeuta, en su formación profesional posee bases conceptuales y prácticas para efectuar un abordaje inicial, sin desconocer que para ello, exige apropiarse de conceptos fundamentales de cultura y de la dinámica de las organizaciones. 


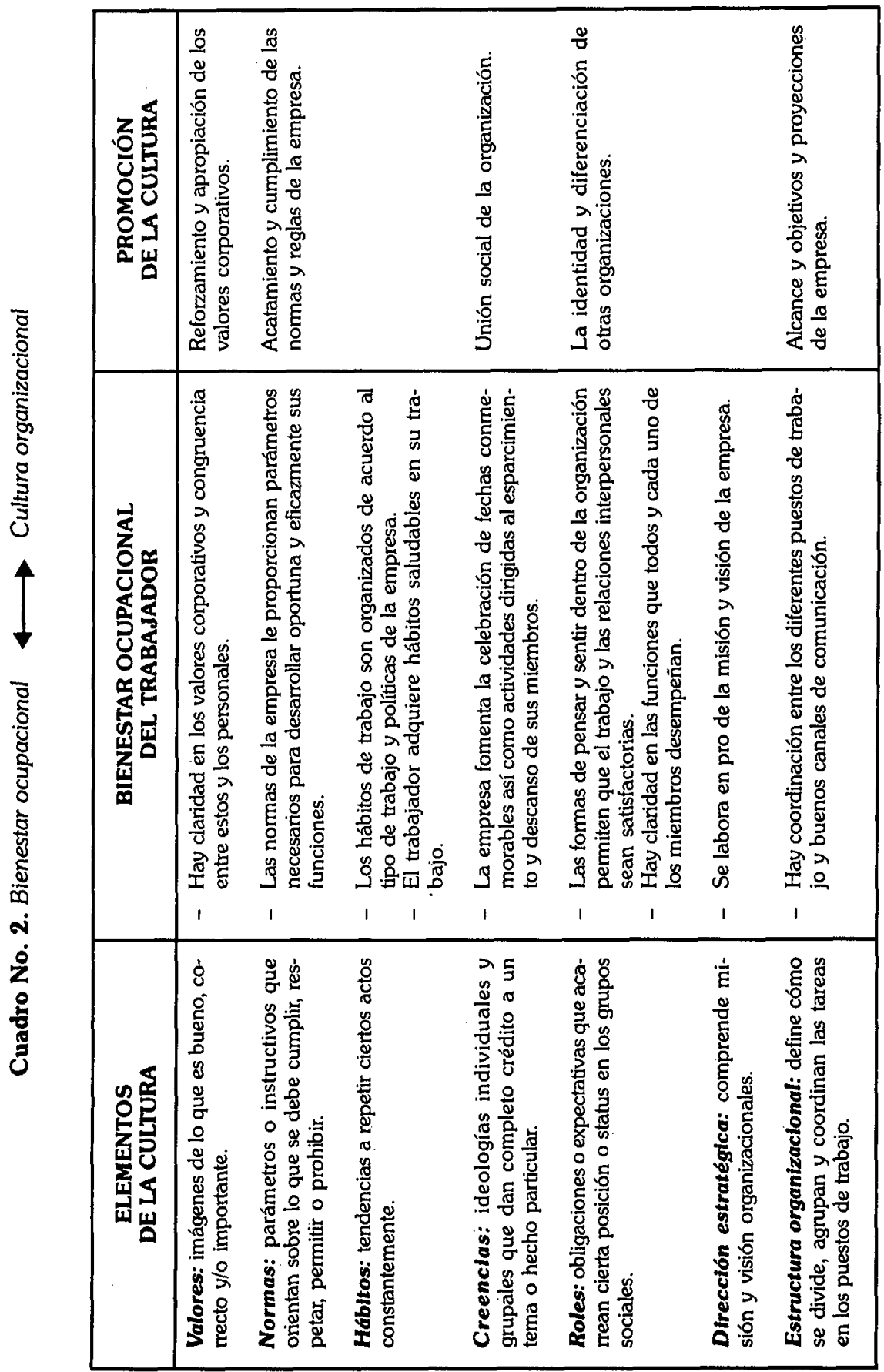




\begin{tabular}{|c|c|}
\hline $\begin{array}{l}\text { Malestar experimentado } \\
\text { por el trabajador } \\
\text { (Torres, 1993) }\end{array}$ & Efectos en la empresa \\
\hline $\begin{array}{l}\text { - Sentimientos de subvaloración e impro- } \\
\text { ductividad. } \\
\text { - No utilización de los recursos necesarios } \\
\text { para la calidad y eficacia de su desempeño. } \\
\text { - Percepción no enriquecedora de su pro- } \\
\text { ceso laboral. } \\
\text { - Acumulación de experiencias negativas. } \\
\text { - No satisfacción de necesidades persona- } \\
\text { les y sociales. } \\
\text { - Impotencia por no poder manifestar el } \\
\text { desacuerdo. } \\
\text { de la empresa que no tiene que ver direc- } \\
\text { tamente con las labores. }\end{array}$ & $\begin{array}{l}\text { - Bajos niveles de producción. } \\
\text { - Ausentismo laboral. } \\
\text { - Insatisfacción del recurso humano. } \\
\text { - Descuido en el manejo de equipos y he- } \\
\text { ramientas que puedan ocasionar acciden- } \\
\text { tes de trabajo y que para la empresa de- } \\
\text { manden gastos económicos. } \\
\text { - Restricción de la participación social. } \\
\text { - Clima laboral tenso. } \\
\text { - Roces y conflictos difíciles de solucionar en } \\
\text { las relaciones interpersonales. } \\
\text { - Excesiva tensión que produce sentimien- } \\
\text { tos agresivos. } \\
\text { - Los trabajadores no se identifican con los } \\
\text { objetivos y políticas de la empresa. }\end{array}$ \\
\hline
\end{tabular}

Cuadro N. 3. Efectos negativos tanto en el trabajador como en la empresa.

\section{Fundamentación teórica}

Desde las bases teóricas o conceptuales, es conveniente profundizar en fundamentos científicos, filosóficos, referentes teóricos y conceptos de cultura y organización.

Los fundamentos científicos se derivan de las ciencias biomédicas, sociales y económicas, donde el terapeuta ocupacional cuenta con una gama de conocimientos provenientes de disciplinas como la psicología, antropología y administración.

En cuanto a los fundamentos filosóficos, éstos representan la visión que guía y da sentido a la profesión (Shannon, citado por Hopkins, 1998), trayendo a consideración los principios que orientan y dirigen la construcción teórica y el suministro de servicios humanos (Trujillo, 1987). 
A partir de éstos, surgen referentes teóricos como los marcos de referencia, que unifican teorías e hipótesis de un área de estudio o práctica.

Con respecto a los conceptos acerca de cultura y de organización, son muchos los fundamentos provenientes de las ciencias Administrativas, Sociales y Humanas, que favorecen conocer cómo los factores genéricos de la cultura influyen en la ocupación.

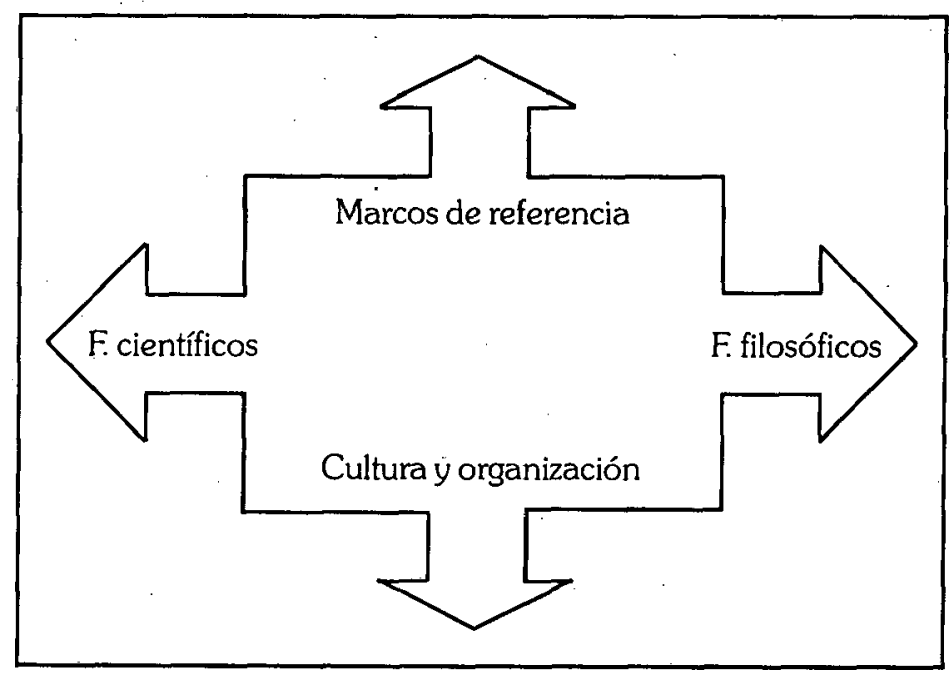

Figura No. 1. Fundamentos teóricos de competencia profesional.

\section{Fundamentación práctica}

En la práctica profesional, el desempeño del terapeuta ocupacional se da mediante un proceso interactivo o dinámico con la organización, a partir de momentos o etapas específicas de intervención y/o participación, señaladas en la figura No. 2. 


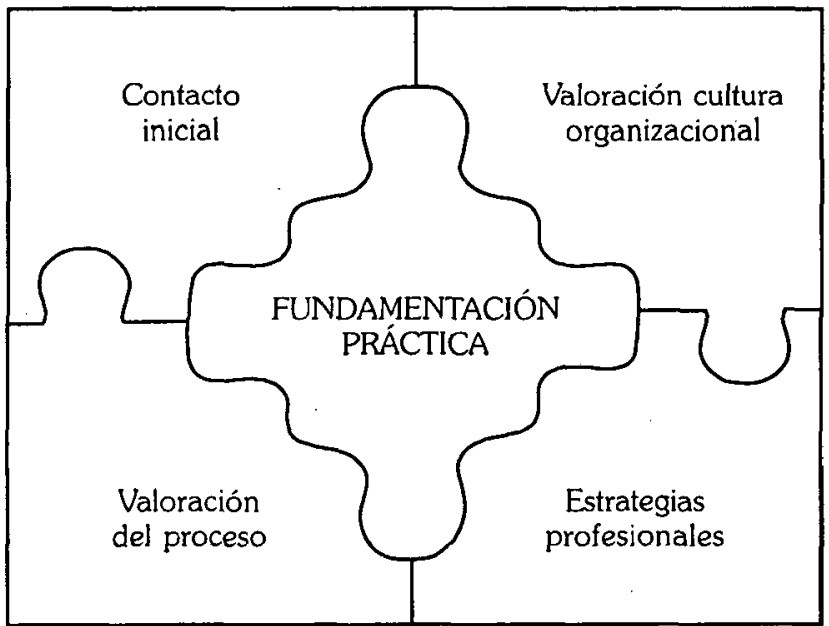

Figura No. 2: Fundamentación práctica de competencia.

1. Contacto inicial: Acercamiento del terapeuta ocupacional a la organización/empresa, con el propósito de establecer procesos de comunicación para la promoción y prestación de sus servicios.

2. Valoración de la cultura organizacional: Procedimiento que permite determinar o comprender la influencia de la cultura en el bienestar ocupacional de los trabajadores, mediante la utilización de instrumentos y técnicas que el terapeuta ocupacional puede emplear o adaptar, asumiendo constantemente los procesos de Escucha, Observación y Participación/actuación, que según Mattingly y Beer (1998), son indispensables para comprenderla.

3. Planeación y ejecución de estrategias profesionales: Se orienta hacia la conservación, mejoramiento o promoción del bienestar ocupacional, de acuerdo a las características de la empresa y a la comprensión cultural de su situación, empleando y a la vez implementando estrategias como:

- Promoción de valores que enriquezcan el trabajo.

- Orientación al trabajador en el uso adecuado de su tiempo.

- Fomento de intereses avocacionales.

- Confrontación de las capacidades del trabajador con las demandas del puesto de trabajo. 
- Identificación y control de riesgos ocupacionales.

- Modificación al ambiente y puesto de trabajo.

- Organización de procesos de interacción y socialización.

4. Evaluación del proceso: Es el procedimiento para determinar el cumplimiento de metas, el eficaz desarrollo del plan de acción y la culminación satisfactoria, en términos de tiempo, resultados y logros con los trabajadores, haciendo uso de los mismos procesos para valorar la cultura empresarial.

\section{CONCLUSIONES}

- El terapeuta ocupacional cuenta con fundamentaciones propias de su profesión que le permiten abordar la dinámica cultural de una empresa. Sin embargo, como todo profesional al dedicarse en un campo específico de su interés, le demanda hacer un recorrido en los saberes que le van a apoyar y fundamentar en una nueva dinámica intelectual.

- El trabajo interdisciplinario en las empresas, favorece la consecución de objetivos orientados a la salud y bienestar de los trabajadores en general, por lo que cobra vital importancia que el terapeuta ocupacional participe colectivamente de este tipo de acciones.

- El estudio por su carácter documental, presenta conceptos que ameritan ser puestos a consideración por parte de la comunidad profesional, para su confrontación y proyección.

\section{LISTA DE REFERENCIAS}

- $\quad$ Allaire, Y. \& Firsirotu, M. (1992). Teorías sobre cultura organizacional. En Abravanel y Cols. (Eds). Cultura Organizacional: Àspectos teóricos, prácticos y metodológicos. (J. Villamizar. Trad.) (pp. 3-37). Colombia: Legis. (Trabajo original publicado en 1982).

- Dyck, I. \& Forwell, S. (1997). Occupational therapy student's first year fieldwork experiences: Discovering the complexity of cultura. Canadian Journal or Occupational Therapy, 64 (4), 185-196. 
- Hocking, C. \& Whiteford, G. (1995). Multiculturalism in Occupational therapy: a time for reflection on core values. Australian Occupational Therapy Journal, 42 (4), 172-175.

- Hopkins, H. (1998). Fundamentos filosóficos de la terapia ocupacional. En H. Hoppkins \& H. Smith (Eds.). (S. Koury y Cols., Trad.). Terapia Ocupacional. ( $8^{\text {a }}$ Ed.). (pp. 58-59). España: Medica Panamericana. (Original publicado en 1993).

- Mac Donald, R. (1998). What is cultural competency? The British Journal of Occupational Therapy, 61 (7). $325-328$.

- Mattingly, C., \& Beer, D. (1998). La interpretación de la cultura en un contexto terapéutico. En H. Hopkins \& H. Smith (Eds). Terapia ocupacional. (S. Koury y cols Trads). (pp.154-161). España: Médica Panamericana S.A. (Trabajo original publicado en 1993).

- Sharon L. Fanelli (1999). Encountering the Concept of Culture in the Heallth Care Setting. The Journal of Occupational Therapy Students. April 1999.

- Torres, M. (1993). El Terapeuta Ocupacional un profesional clave en el Bienestar del Trabajador y la Productividad de la Empresa. Revista Ocupación Humana, 5 (2), $35-38$

- Trujillo, A. (1987). Reflexiones sobre la identidad del Terapeuta Ocupacional. Revista Acción, 2, (1), 33-47.

- Uribe, L.; Rodríguez, E. \& Acosta, M. (1999). Competencia del terapeuta ocupacional en el abordaje de la cultura organizacional. Tesis profesional, Universidad Nacional de Colombia. 